\title{
A STRUCTURAL PROPERTY OF ADIAN INVERSE SEMIGROUPS
}

\author{
MUHAMMAD INAM, JOHN MEAKIN, AND ROBERT RUYLE \\ Abstract. We prove that an inverse semigroup over an Adian presen- \\ tation is E-unitary.
}

Dedicated to the memory of John M. Howie

\section{INTRODUCTION}

Throughout this paper, $X$ is a non-empty set and $R=\left\{\left(u_{i}, v_{i}\right): i \in I\right\}$ where $u_{i}, v_{i} \in X^{+}$. We refer to the pair $P=(X, R)$ as a positive presentation. The semigroup with the set $X$ of generators and the set $R$ of relations will be denoted by $S g p\langle X \mid R\rangle$ and the corresponding group will be denoted by $G p\langle X \mid R\rangle$. We obtain two undirected graphs corresponding to $P$ as follows. The left graph $L G(P)$ of $P$ has set $X$ of vertices and has an undirected edge connecting $x$ and $y$ in $X$ corresponding to each relation of the form $\left(u_{i}, v_{i}\right)$ in $R$ where $x$ is the first letter of $u_{i}$ and $y$ is the first letter of $v_{i}$. The right graph $R G(P)$ is constructed dually, with edges connecting the last letters of pairs $\left(u_{i}, v_{i}\right)$ in $R$. If there is no cycle in either the left graph or the right graph of a positive presentation, then the presentation is called a cycle-free presentation or an Adian presentation. These presentations were first studied by S. I. Adian in [1], where it was shown that the semigroup $S g p\langle X \mid R\rangle$ embeds in the group $G p\langle X \mid R\rangle$. Unless stated otherwise, we will consider our presentations to be Adian presentations throughout.

A semigroup $S$ is called an inverse semigroup if for each $a \in S$ there exists a unique element $b \in S$ such that $a b a=a$ and $b a b=b$. This unique element $b$ is denoted by $a^{-1}$. It is well known that idempotents commute in an inverse semigroup and that the product of two idempotents is an idempotent. If $S$ is an inverse semigroup, then the natural partial order on $S$ is defined, for $a, b \in S$, by $a \leq b$ if and only if $a=a a^{-1} b$. A congruence relation $\sigma$ is defined on $S$, for $a, b \in S$, by $a \sigma b$ if and only if there exists an element $c \in S$ such that $c \leq a, b$. Then $\sigma$ is the minimum group congruence relation on $S$, i.e., $S / \sigma$ is the maximum group homomorphic image of $S$. We refer the reader to the text [2] for these facts and further details about inverse semigroups. The following proposition concerning idempotents and the natural partial order is standard in the literature on inverse semigroups; we will use these facts often in this paper.

Date: November 13, 2015. 
Proposition 1. Let $S$ be an inverse semigroup.

(i) If $e$ is an idempotent and $a \leq e$ in $S$, then $a$ is an idempotent.

(ii) If $x \in S$ and $e$ is an idempotent of $S$, then $x e x^{-1}$ is an idempotent.

(iii) Suppose $x_{0}, \ldots, x_{n} \in S$ and suppose $e_{1}, \ldots, e_{n}$ are idempotents. Then $x_{0} e_{1} x_{1} \cdots e_{n} x_{n} \leq x_{0} x_{1} \cdots x_{n}$. In particular, if $x_{0} x_{1} \cdots x_{n}$ is an idempotent, then $x_{0} e_{1} x_{1} \cdots e_{n} x_{n}$ is an idempotent.

Inverse semigroups form a variety of algebras in the sense of universal algebra, and so free inverse semigroups exist. The inverse semigroup with the set $X$ of generators and the set $R$ of relations is denoted by $\operatorname{Inv}\langle X \mid R\rangle$. This is the quotient of the free inverse semigroup on $X$ obtained by imposing the relations $u_{i}=v_{i}$ in $R$. See Stephen's paper [5] for basic information about presentations of inverse semigroups. A basic fact is that if an inverse semigroup $S$ is given by a presentation $S=\operatorname{Inv}\langle X \mid R\rangle$ then the group $S / \sigma$ is given by the presentation $G p\langle X \mid R\rangle$.

An inverse semigroup $S$ is called E-unitary if the natural homomorphism from $S$ onto $G=S / \sigma$ is idempotent-pure, i.e., if the inverse image of the identity of $G$ is equal to the set of idempotents of $S$. We refer to [2] for equivalent definitions and further information about the importance of $E$ unitary inverse semigroups in the literature on inverse semigroups.

In this paper we prove that if $P=(X, R)$ is an Adian presentation, then the inverse semigroup $S=\operatorname{Inv}\langle X \mid R\rangle$ is E-unitary.

\section{Main TheOrem}

In order to set the foundations of our main theorem, we first give a common definition of a van Kampen diagram over a presentation $(X, R)$ and then describe some properties of van Kampen diagrams over an Adian presentation $(X, R)$.

A van Kampen diagram $\Delta$ is a planar, finite, connected, simply connected, oriented 2-complex (often called a "map"). There is an involution $e \rightarrow e^{-1}$ on the edges of the diagram such that $\left(e^{-1}\right)^{-1}=e$ and $e^{-1} \neq e$. The pair $\left\{e, e^{-1}\right\}$ may be considered as an undirected edge with one of these edges viewed as positively oriented and the other as negatively oriented. A van Kampen diagram over the presentation $(X, R)$ satisfies:

(i) Each directed positively oriented edge of the 1-skeleton is labeled by a letter in $X$ and its inverse is labeled by the inverse of that letter.

(ii) The 2-cells (sometimes called "regions" or "faces") are homeomorphic to open disks. The boundary of each 2 -cell is labeled by a cyclically reduced conjugate of $u v^{-1}$ for some relation $(u, v) \in R$.

(iii) There is a distinguished base vertex $O$ that lies on the topological boundary of $\Delta$.

The boundary cycle of $\Delta$ that begins and ends at the base vertex $O$, and travels in the counterclockwise direction around the boundary, is labeled by some (not necessarily freely reduced) word $w \in\left(X \cup X^{-1}\right)^{*}$. In this case, we say that $\Delta$ is a van Kampen diagram for $w$ over $(X, R)$. 
A van Kampen diagram $\Delta$ is said to be reduced if it satisfies the additional condition:

(iv) There do not exist two 2-cells with an edge in common to their boundaries that are mirror images of each other.

In this paper we will be working mainly with reduced van Kampen diagrams. The well known Lemma of van Kampen says: (1) The boundary label of a reduced van Kampen diagram over $(X, R)$ is a word $w$ that is equal to the identity in $G=G p\langle X \mid R\rangle$. (2) For every every word $w$ representing the identity of $G$ there exists a reduced van Kampen diagram that has $w$ as its boundary label.

We remark that some authors require a van Kampen diagram to have a reduced word as its boundary label, and in fact some require the boundary label to be a cyclically reduced word. It is convenient for our purposes to make no such restriction however. We refer to the book by Lyndon and Schupp [3] for more detail and for basic information about van Kampen diagrams. Such diagrams are referred to as g-diagrams in the paper of Remmers [4]. A $g$-diagram in Remmers' sense is required to have a cyclically reduced word as boundary label, but this restriction is in fact not essential.

Remark 1. It follows from van Kampen's Lemma that the inverse semigroup $S=\operatorname{Inv}\langle X \mid R\rangle$ is E-unitary if and only if, for every van Kampen diagram $\Delta$ over $(X, R)$, the word $w$ labeling the boundary cycle of $\Delta$ that starts and ends at the distinguished vertex 0 is an idempotent of $S$.

Each 2-cell of a van Kampen diagram over an Adian presentation $P=$ $(X, R)$ is two-sided. The boundary of a such a 2-cell can be viewed as a birooted graph, where one side of a relation $(u, v) \in R$ labels a path $p$ from the initial vertex to the terminal vertex of the graph, while the other side of the relation labels a different path $q$ from the initial vertex to the terminal vertex of the graph. We refer to these paths as the sides of the 2-cell. The path $p q^{-1}$ is a boundary cycle of the 2-cell.

A vertex $\alpha$ of a van Kampen diagram $\Delta$ is called a source if all edges of $\Delta$ with initial vertex $\alpha$ are positively labeled. Sinks of $\Delta$ are defined dually. The following fact about van Kampen diagrams over Adian presentations was proved by Remmers [4] (Theorem 4.3) for diagrams whose boundary label is a cyclically reduced word: however, Remmers' proof carries over verbatim for the more general notion of van Kampen diagrams that we are considering in this paper.

Lemma 1. Let $\Delta$ be a reduced van Kampen diagram for a word $w$ over an Adian presentation $(X, R)$. Then $\Delta$ has no interior sources and no interior sinks.

The following lemma follows from the above discussion and from Lemma 2.2 and Theorem 4.3 of [4].

Lemma 2. If $\Delta$ is a reduced van Kampen diagram over an Adian presentation, then $\Delta$ satisfies the following conditions: 
(i) Every 2-cell of $\Delta$ is two-sided.

(ii) $\Delta$ contains no directed (i.e., positively labeled) cycles.

(iii) Every positively labeled interior edge of $\Delta$ can be extended to a directed transversal of $\Delta$.

Here, by a directed transversal of $\Delta$ we mean a positively labeled path between two distinct boundary vertices of $\Delta$, all of whose vertices are distinct, all of whose edges are interior to $\Delta$, and all of whose vertices except the initial and terminal vertex are interior vertices in $\Delta$.

The following result is the main theorem of the paper.

Theorem 2.1. An inverse semigroup $S=\operatorname{Inv}\langle X \mid R\rangle$ over an Adian presentation is E-unitary.

Before proving this theorem, we introduce some definitions and prove some lemmas.

Definiton 1. A subdiagram $\Delta^{\prime}$ of a van Kampen diagram $\Delta$ is called a simple component of $\Delta$ if it is a maximal subdiagram whose boundary is a simple closed curve.

We remark that our definition does not require that the boundary of a simple component of reduced diagram $\Delta$ needs to be labeled by a reduced word. However, since no 2-cell of a van Kampen diagram over an Adian presentation has a boundary that contains an extremal vertex (i.e. a vertex of degree 1), it follows that a simple component of such a diagram does not contain any attached trees as part of its boundary. A simple component of $\Delta$ is itself a van Kampen diagram. The diagram $\Delta$ has a tree-like (or "cactoid") structure of simple components connected by (possibly trivial) arcs and possibly with finitely many finite trees attached to the boundary of the diagram (see Figure 1). A van Kampen diagram with no simple components is just a finite (edge-labeled) tree.

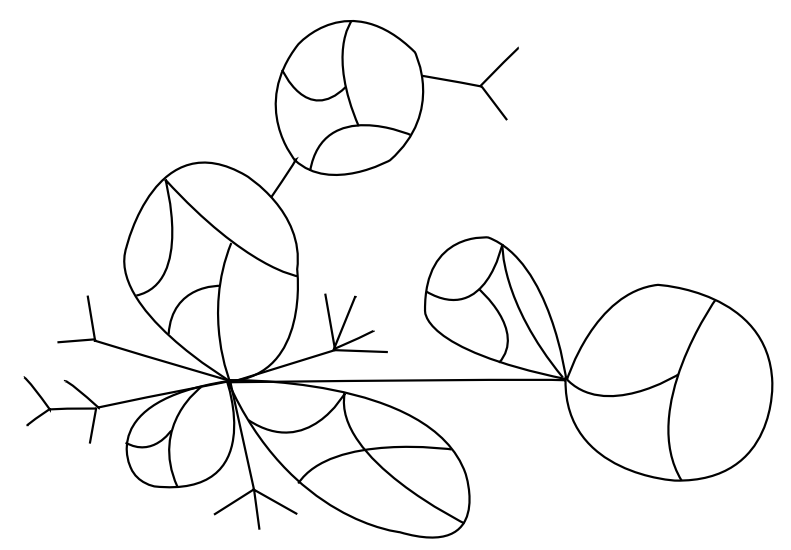

FiguRe 1. 
Definiton 2. For a van Kampen diagram $\Delta$ over an Adian presentation $(X, R)$, a transversal subdiagram $\Delta^{\prime}$ is a subdiagram of a simple component of $\Delta$ such that $\Delta^{\prime}$ has a boundary cycle of the form $p q$, where $p$ is a directed transversal and $q$ is a subpath of a boundary cycle of the simple component in which $\Delta^{\prime}$ is contained.

Lemma 3. If $\Delta$ is a van Kampen diagram with exactly one simple component and no extremal vertex, then $\Delta$ has a directed transversal if and only if it has more than one 2-cell. Furthermore, any directed transversal of $\Delta$ divides $\Delta$ into two transversal subdiagrams, each of which may be viewed as a van Kampen diagram with exactly one simple component and no extremal vertex.

Proof. If $\Delta$ has a directed transversal, then it must have an interior edge and hence it must have more than one 2-cell. Conversely if $\Delta$ has more than one 2-cell and has just one simple component, it must have an interior edge $e$. We extend this edge to a directed transversal $T$ of $\Delta$ by using Lemma 2 above. Denote the initial vertex of $T$ by $\alpha$ and the terminal vertex of $T$ by $\beta$. Thus $\alpha$ and $\beta$ are on the boundary of $\Delta$. This transversal $T$ divides $\Delta$ into two proper subdiagrams $\Delta_{1}$ and $\Delta_{2}$ (see Figure 2). Both $\Delta_{1}$ and $\Delta_{2}$ are clearly transversal subdiagrams of $\Delta$. Also, the boundary of $\Delta$ is a simple closed curve, and $T$ is a path between distinct boundary vertices of $\Delta$ with all of its vertices distinct and all of its vertices except $\alpha$ and $\beta$ interior to $\Delta$. It follows that the boundary of each subdiagram $\Delta_{i}$ is topologically a simple closed curve. Hence each subdiagram $\Delta_{i}$ may be viewed as a van Kampen diagram with exactly one simple component and no extremal vertex.

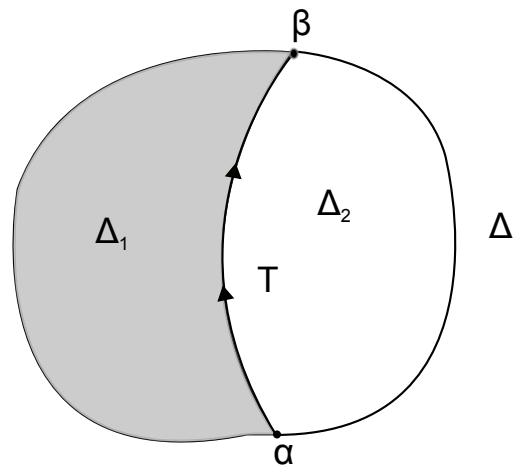

Figure 2. A diagram $\Delta$ with two transversal subdiagrams.

Definiton 3. For a van Kampen diagram $\Delta$ over an Adian presentation $(X, R)$, a special 2-cell is a 2-cell, one of whose two sides lies entirely on the boundary of $\Delta$. 
Lemma 4. Let $\Delta$ be a van Kampen diagram over an Adian presentation $(X, R)$ that has more than one 2-cell and that has just one simple component and no extremal vertex. Then $\Delta$ contains at least two special 2-cells.

Proof. As in the proof of Lemma 3 we may choose a directed transversal $T$ from a vertex $\alpha$ on $\partial \Delta$ to a vertex $\beta$ on $\partial \Delta$ that divides the diagram $\Delta$ into two subdiagrams $\Delta_{1}$ and $\Delta_{2}$.

If both of the subdiagrams $\Delta_{1}$ and $\Delta_{2}$ consist of only one 2-cell, then we are done. Otherwise, we repeatedly subdivide each of these subdiagrams to find special 2-cells of $\Delta$. Without loss of generality, we pick $\Delta_{1}$ and find an interior edge $e_{1}$ in $\Delta_{1}$. We extend $e_{1}$ to a directed transversal $T_{1}$ of $\Delta_{1}$. Let $\alpha_{1}$ be the initial vertex of $T_{1}$ and $\beta_{1}$ the terminal vertex of $T_{1}$. This transversal $T_{1}$ divides $\Delta_{1}$ into two proper subdiagrams. We claim that at least one of them is a transversal subdiagram of $\Delta$. To see this we consider the following four cases.

Case 1: Suppose $\alpha_{1}$ and $\beta_{1}$ lie on the boundary $\partial \Delta_{1} \cap \partial \Delta$. Since $T_{1}$ is a positively labeled path, the collection of 2-cells and their boundaries bounded by the path $T_{1}$ and the path from $\beta_{1}$ to $\alpha_{1}$ along $\partial \Delta_{1} \cap \partial \Delta$ forms a transversal subdiagram of $\Delta$, which contains fewer 2-cells than $\Delta_{1}$ (see Figure 3).

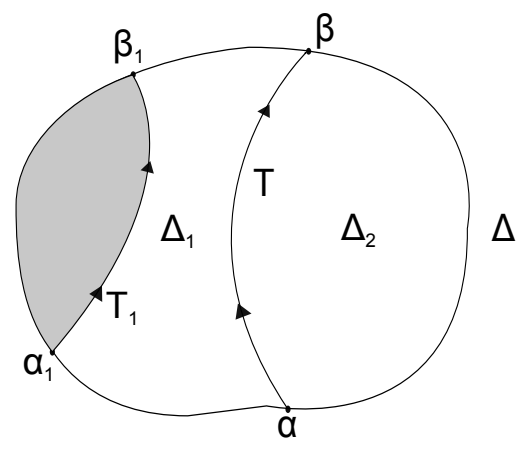

FiguRE 3.

Case 2: Suppose both $\alpha_{1}$ and $\beta_{1}$ lie on the transversal $T$. Note that if the transversals $T$ and $T_{1}$ are oppositely oriented, then our van Kampen diagram $\Delta$ contains a directed (positively labeled) cycle, which contradicts Lemma 2 above. Hence both transversals will have the same orientation. Let $T_{2}$ be the composition of the path along $T$ from $\alpha$ to $\alpha_{1}$ followed by the path $T_{1}$, followed by the path along $T$ from $\beta_{1}$ to $\beta$ (see Figure 4 ). Clearly $T_{2}$ is a positively labeled path that is a transversal of $\Delta$.

The collection of 2-cells and their boundaries bounded by $T_{2}$ and the path that goes from $\beta$ to $\alpha$ along $\partial \Delta_{1} \cap \partial \Delta$ forms a transversal subdiagram of $\Delta$ that contains fewer 2-cells than $\Delta_{1}$.

Case 3: Suppose $\alpha_{1}$ lies on the boundary $\partial \Delta_{1} \cap \partial \Delta$ and $\beta_{1}$ lies on the path $T$. Let $T_{2}$ be the composition of the path $T_{1}$ and the path along $T$ 

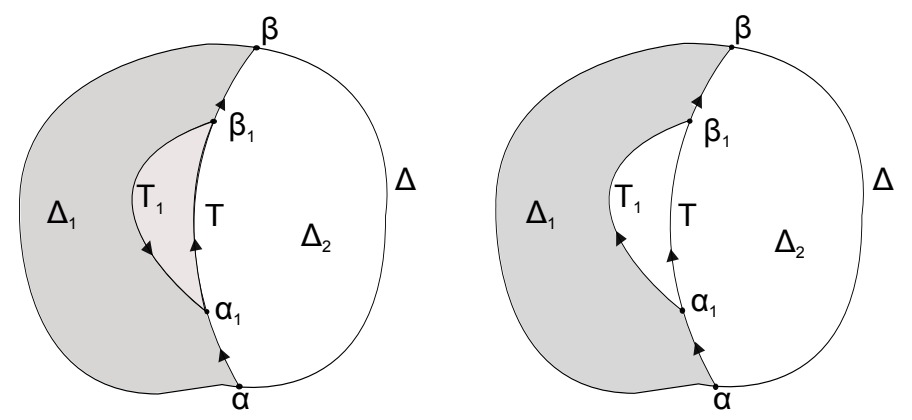

FIGURE 4.

from $\beta_{1}$ to $\beta$ (see Figure 5). Again, $T_{2}$ is a positively labeled path that is a transversal of $\Delta$. The collection of 2 -cells bounded by $T_{2}$ and the path from $\beta$ to $\alpha_{1}$ along $\partial \Delta_{1} \cap \partial \Delta$ forms a transversal subdiagram of $\Delta$ that contains fewer regions than $\Delta_{1}$.

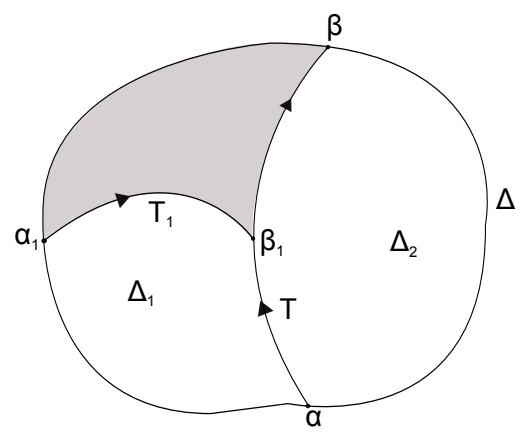

FiguRe 5.

Case 4: Suppose that $\alpha_{1}$ lies on the path $T$ and $\beta_{1}$ lies on the boundary $\partial \Delta_{1} \cap \partial \Delta$. In this case let $T_{2}$ be the composition of the path along $T$ from $\alpha$ to $\alpha_{1}$ and the path $T_{1}$ (see Figure 6). Then the collection of 2-cells bounded by $T_{2}$ and the path from $\beta_{1}$ to $\alpha$ along $\partial \Delta_{1} \cap \partial \Delta$ forms a transversal subdiagram of $\Delta$ that contains fewer 2-cells than $\Delta_{1}$.

Since our original diagram has only finitely many 2-cells, this process eventually terminates and we get a minimal transversal subdiagram $D$ of $\Delta$ inside $\Delta_{1}$ that consists of only one 2-cell. This 2 -cell $D$ is bounded by a cycle $p q$ where $p$ is a directed transversal of $\Delta_{1}$ and $q$ is a path along the boundary of $\Delta$. Since $D$ is a 2 -cell, it has a boundary label of the form $u v^{-1}$ for some relation $(u, v) \in R$. Since $p$ is positively labeled, neither the initial vertex nor the terminal vertex of the 2-cell $D$ can lie on $p$ (or else the orientation of $p$ would change at that vertex). Hence both the initial and terminal vertices of $D$ lie on the boundary of $\Delta$, and so $D$ is a special 2-cell of $\Delta$. A similar argument yields a special 2-cell of $\Delta$ inside $\Delta_{2}$. 


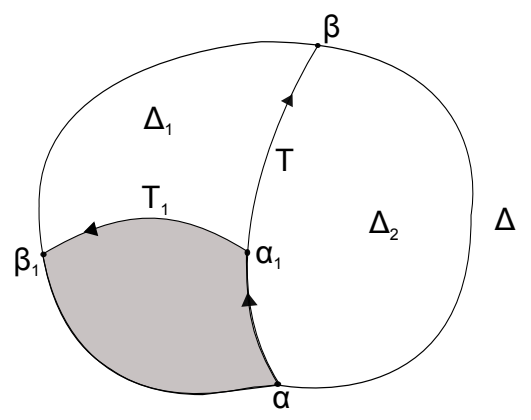

FiguRe 6.

Remark 2. We remark for future use that by Lemma 3 , the directed transversal $p$ constructed in the proof of Lemma 4 above divides $\Delta$ into two subdiagrams, one of which is the special 2-cell $D$ and the other of which has just one simple component.

Lemma 5. If $\Delta$ is a van Kampen diagram over an Adian presentation $P=(X, R)$ and $\Delta$ has just one simple component and no extremal vertex, then any word labeling a boundary cycle of $\Delta$, starting and ending at any vertex 0 on $\partial \Delta$, is an idempotent in the inverse monoid $S=\operatorname{Inv}\langle X \mid R\rangle$.

Proof. We apply induction on the number of 2-cells of $\Delta$. If $\Delta$ contains only one 2-cell, then a boundary label of this 2-cell is a cyclic conjugate of $u v^{-1}$, for some $(u, v) \in R$. We know that $u v^{-1}$ is an idempotent in $S$, because in the inverse semigroup $S, u v^{-1}=v v^{-1}$. We show that any cyclic conjugate of $u v^{-1}$ is also an idempotent in $S$. Suppose that $u$ factors as a product of two subwords $u \equiv x y$ in $X^{+}$. We consider the word $y v^{-1} x$ and show that it is an idempotent in $S$. Note that $y v^{-1} x=y u^{-1} x=y(x y)^{-1} x=y y^{-1} x^{-1} x$, a product of two idempotents in $S$, hence it is an idempotent in $S$. We remark that it follows that the inverse of this word, namely $x^{-1} v y^{-1}$, is also an idempotent of $S$ (in fact it equals $y v^{-1} x$ in $S$ ), so all boundary labels of the 2-cell $\Delta$ are idempotents in $S$, no matter which orientation of the boundary of $\Delta$ is chosen.

Suppose the conclusion of the lemma is true for all van Kampen diagrams consisting of one simple component and $k 2$-cells. Let $\Delta$ be a van Kampen diagram consisting of one simple component and $(k+1) 2$-cells. Then by Lemma 4, there are at least two special 2-cells in $\Delta$. These special 2-cells do not share an edge that lies on $\partial \Delta$. So at least one of them (say the 2-cell $\Pi)$, has the property that the distinguished vertex 0 is an initial or terminal vertex of at least one edge in $\partial \Delta-\partial \Pi$ (i.e. it does not lie in the "interior" of $\partial \Pi \cap \partial \Delta)$.

The cell $\Pi$ has a boundary label $u v^{-1}$ for some $(u, v) \in R$, such that either $u$ or $v$ labels a path on $\partial \Delta$. Without loss of generality, we assume that $u$ labels a path on $\partial \Delta$. Denote the initial vertex of this path by $\alpha$ and the terminal vertex by $\beta$. By Remark 2, we may suppose that $v=s r t$ where 
$s$ labels a path on the boundary of $\Delta$ from $\alpha$ to some vertex $\alpha_{1}, r$ labels a directed transversal from $\alpha_{1}$ to $\beta_{1}$, and $t$ labels a path on the boundary of $\Delta$ from $\beta_{1}$ to $\beta$ (see Figure 7). The distinguished vertex 0 of the van Kampen diagram $\Delta$ does not lie in the interior of the part of the boundary of $\Delta$ that is labeled by $t u^{-1} s$. Hence the label on the boundary cycle of $\partial \Delta$ that starts and ends at 0 is of the form $\ell(\partial \Delta) \equiv g s^{-1} u t^{-1} h$ for some words $g, h \in\left(X \cup X^{-1}\right)^{*}$. Then in the inverse semigroup $S, g s^{-1} u t^{-1} h=$ $g s^{-1} v t^{-1} h=g s^{-1} s r t t^{-1} h \leq g r h$, by Proposition 1. But by Lemma 3 and Remark 2, grh labels a boundary cycle of a van Kampen diagram with only one simple component and fewer 2-cells than $\Delta$. Hence this word is an idempotent in $S$ by the induction hypothesis. Hence $\ell(\partial \Delta) \equiv g s^{-1} u t^{-1} h$ is an idempotent of $S$, again by Proposition 1. As we remarked before, if we choose the opposite orientation on the boundary of $\Delta$, the corresponding boundary label is also an idempotent of $S$.

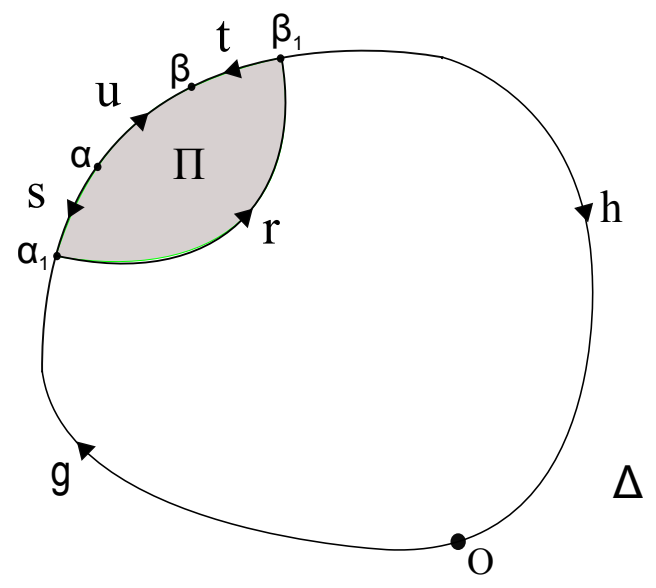

FiguRe 7.

Corollary 1. If $\Delta$ is a van Kampen diagram over an Adian presentation $P=(X, R)$ that has just one simple component, then any word labeling a boundary cycle of $D$, starting and ending at any vertex 0 on $\partial D$, is an idempotent in the inverse monoid $S=\operatorname{Inv}\langle X \mid R\rangle$.

Proof. The van Kampen diagram $\Delta$ either has no extremal vertex, in which case the result follows from Lemma 5 or else is obtained from a van Kampen diagram $\Delta^{\prime}$ with no extremal vertex and just one simple component by adjoining finitely many finite trees to the boundary of $\Delta^{\prime}$. The result follows from Proposition 1 and the fact that the label on a boundary cycle of $\Delta$ is obtained from the label $w$ on a boundary cycle of $\Delta^{\prime}$ either by inserting Dyck words (words with reduced form 1 , which are idempotents in $S$ ), or if necessary conjugating the resulting word by some word $u$ if the distinguished vertex 0 is on an attached tree. 
Proof of Theorem 2.1. Let $(X, R)$ be an Adian presentation and let $w$ be a word such that $w=1$ in $G=G p\langle X \mid R\rangle$. Then $w$ labels a boundary cycle of a van Kampen diagram $\Delta$ starting and ending at some designated vertex 0 on $\partial \Delta$. We show that $w$ is an idempotent of $S=\operatorname{Inv}\langle X \mid R\rangle$ by induction on the number of simple components of $\Delta$. If $\Delta$ has just one simple component, this follows from Corollary 1. Of course if $D$ has no simple components, then it is a finite tree, whose boundary label is a Dyck word, which is an idempotent of $S$.

Suppose that $\Delta$ has $k>1$ simple components. Assume inductively that the word labeling a boundary cycle of any van Kampen diagram with fewer than $k$ simple components, starting and ending at any vertex on its boundary, is an idempotent in $S$.

It follows from the fact that $\Delta$ is simply connected that $\Delta$ has a cut vertex $\gamma$, i.e., a vertex on the boundary of $\Delta$ whose deletion separates $\Delta$ into two or more connected components $K_{1}, K_{2}, \ldots K_{n}$. For each $i$, let $D_{i}$ be the 2-complex $D_{i}=K_{i} \cup\{\gamma\}$. Then each $D_{i}$ is a van Kampen diagram over $(X, R)$. The cut vertex can be chosen so that each subdiagram $D_{i}$ has fewer simple components than $\Delta$ (see Figure 8).

Then $\Delta=D_{1} \vee D_{2} \vee \ldots \vee D_{n}$ is obtained by joining the diagrams $D_{i}$ at the vertex $\gamma$.

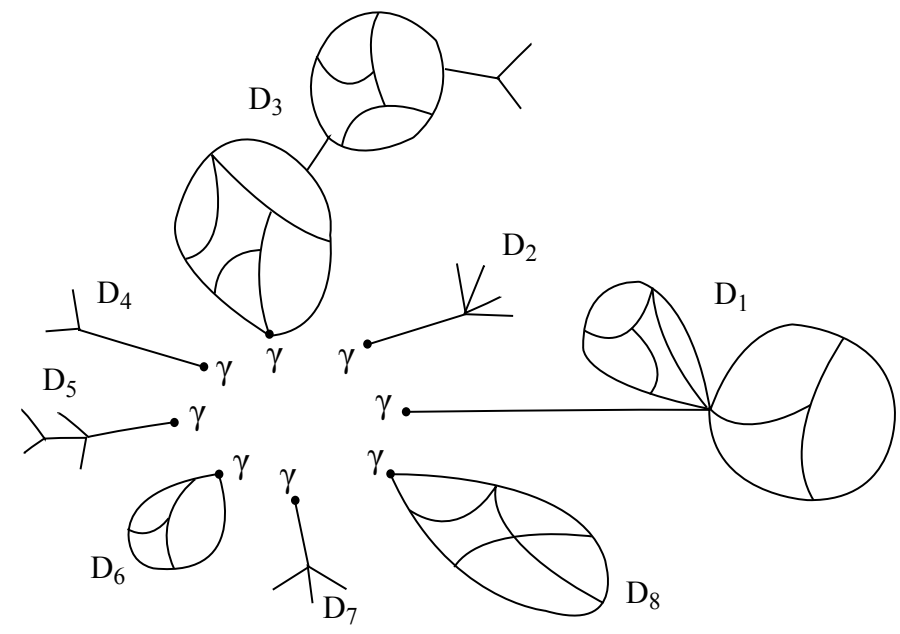

FiguRe 8.

Let $s_{i}=\ell\left(q_{i}\right)$ be the label on the boundary cycle $q_{i}$ around $D_{i}$ that starts and ends at $\gamma$ and respects the orientation of the boundary cycle $p$ of $\Delta$ labeled by $w$. Then $s_{i}$ is an idempotent of $S$ by the induction hypothesis.

If $0=\gamma$, then the boundary cycle $p$ is labeled by some permutation of the word $s_{1} s_{2} \ldots s_{n}$, a product of (commuting) idempotents in $S$. Therefore in $S, w=s_{1} s_{2} \ldots s_{n}$, and hence $w$ is an idempotent in $S$. 
If 0 is not equal to $\gamma$, then it must be a vertex on the boundary of exactly one of the van Kampen diagrams $D_{i}$. Let $s_{i} \equiv x_{i} y_{i}$ where $x_{i}$ labels the portion of the boundary cycle $q_{i}$ of $D_{i}$ starting at $\gamma$ and ending at 0 , and $y_{i}$ labels the portion of $q_{i}$ starting at 0 and ending at $\gamma$ in the orientation of $q_{i}$. Then the boundary cycle $p$ of $\Delta$ labeled by $w$ is equal in $S$ to $y_{i}\left(\Pi_{j \neq i} s_{j}\right) x_{i}$, where the product $\Pi_{j \neq i} s_{j}$ is an idempotent of $S$. Hence $w=y_{i}\left(\Pi_{j \neq i} s_{j}\right) x_{j} \leq$ $y_{i} x_{i}$ in $S$, by Proposition 1. But by the induction assumption, $y_{i} x_{i}$ is an idempotent of $S$ since it is the label of a boundary cycle of $D_{i}$ starting at 0 . It follows that the word $w$ is an idempotent in $S$, again by Proposition 1 .

Hence $S$ is $E$-unitary since the minimum group congruence $\sigma$ on $S$ is idempotent-pure.

We close the paper by showing that inverse monoids naturally associated with Adian inverse semigroups of the type considered above are also $E$ unitary. We first record a general fact about positive presentations of inverse semigroups.

Proposition 2. Let $S=\operatorname{Inv}\langle X \mid R\rangle$ be a positive presentation of an inverse semigroup, where $R=\left\{\left(u_{i}, v_{i}\right) \mid i \in I\right\}$ and $u_{i}, v_{i} \in X^{+}$. Then if $S$ is $E$ unitary, so is the inverse monoid $M=\operatorname{Inv}\left\langle X \mid u_{i} v_{i}^{-1}=1, i \in I\right\rangle$.

Proof. Clearly both inverse semigroups $S$ and $M$ have the same maximal group homomorphic image $G=G p\langle X \mid R\rangle$.

If $u_{i} v_{i}^{-1}=1$ in $M$, then $v_{i}=u_{i}\left(v_{i}^{-1} v_{i}\right) \leq u_{i}$ in $M$. Also $v_{i} u_{i}^{-1}=1$, which implies by the same argument that that $u_{i} \leq v_{i}$. Hence $u_{i}=v_{i}$ in $M$. It follows from a standard argument that the natural map from $S$ onto $G$ factors through $M$. Hence if $\sigma$ and $\sigma_{1}$ denote the natural maps from $S$ and $M$ onto $G$ respectively, the fact that $\sigma$ is idempotent-pure implies that $\sigma_{1}$ is idempotent-pure, and so $M$ is $E$-unitary.

Corollary 2. If $\langle X \mid R\rangle$ is an Adian presentation where $R=\left\{\left(u_{i}, v_{i}\right) \mid i \in I\right\}$, then $M=\operatorname{Inv}\left\langle X \mid u_{i} v_{i}^{-1}=1, i \in I\right\rangle$ is E-unitary.

Proof. This is immediate from Theorem 2.1 and Proposition 2.

\section{REFERENCES}

[1] S. I. Adian, Defining relations and algorithmic problems for groups and semigroups, Proc. Steklov Inst. Math., No 85 (1966).

[2] Mark V. Lawson, Inverse semigroups, World Scientific Co. Pte. Ltd., 1998.

[3] R. C. Lyndon and P.Schupp, Combinatorial Group Theory, Springer-Verlag, Heidelberg, 1977.

[4] John H. Remmers, On the Geometry of Semigroup Presentations, Advances in Mathematics, 36, (1980), 283-296.

[5] J. B. Stephen, Presentations of inverse monoids, Journal of Pure and Applied Algebra (1990), 81-112. 
E-mail address: s-minam1@math.unl.edu

E-mail address: jmeakin@math.unl.edu

E-mail address: rruyle3@unl.edu

Department of Mathematics, University of Nebraska-Lincoln, Lincoln, NE 68588, USA 